\title{
Otorrhoea - a parotid connection
}

\author{
J Joseph, (iD S Maharaj iD \\ Department of Otorhinolaryngology, University of the Witwatersrand, Johannesburg, South Africa
}

Corresponding author,email: shivesh.maharaj@wits.ac.za

\begin{abstract}
Summary
A 36-year-old patient presented with an 18-month history of intermittent right parotid swelling accompanied by otorrhoea. The symptoms started subsequent to a palm leaf injury to the right ear. At right parotidectomy, a fistula connecting the right external auditory canal and the right parotid was demonstrated. A small fibrotic mass probably due to a remnant of the palm leaf was found intraoperatively. The symptoms resolved completely after the mass was excised by superficial parotidectomy, and the fistula closed spontaneously.

Keywords: parotid, otorrhea, trauma
\end{abstract}

\section{Case presentation}

A 36-year-old woodcutter presented with an 18-month history of intermittent episodes of right parotitis and otorrhoea accompanied by swelling of the right cheek. The complaints started shortly after he was struck on the right ear by the sharp end of a falling palm leaf. He reported bleeding from the ear at that time and the palm leaf spike was pulled out by his colleagues. On examination, he had a mildly tender swelling of the right parotid. The tympanic membrane was intact and the middle ear was healthy. Radiological investigations (CT scan) showed a collection in the right parotid and a small mass in the superficial lobe of the parotid, and a decision was made to do a superficial parotidectomy. (Figures 1 and 2)

Intraoperatively, a fistula from the anterior part of the external auditory canal leading into the right parotid gland was identified. The fistula led directly to a dense fibrotic mass which was the result of a foreign body reaction to a piece of the palm leaf being embedded in the parotid gland. The mass was entwined with the main trunk of the facial nerve. The parotidectomy along with removal of the fibrotic mass was done in two staged procedures. (Figure 3) The fistula leading into the external auditory canal (EAC) closed spontaneously. Postoperatively, the patient healed well with no facial nerve fallout.

\section{Discussion}

Traumatic injuries to the parotid gland are common. This is due to the fairly superficial location of the gland. The commonest sequelae of parotid injuries are fistulae and sialocoeles. ${ }^{1}$ In a literature review of 70 cases of parotid injuries, 54\% resolved on conservative management. In this particular case, the otorrhoea was an unusual presentation. The key point to note is that the otorrhoea was accompanied by right parotid swelling. There was no history of sialotorrhoea (salivary discharge from the ear during mastication) in this case.

Fistulae can occur as a result of injury to the parotid duct to the gland itself. ${ }^{2}$ If the parotid duct is incorrectly sutured then a fistula to the EAC can develop. ${ }^{2}$ Foramen of Huschke are dehiscences in the EAC which form a natural pathway of spread of infection to the superficial lobe of the parotid gland and vice versa. These can also present as spontaneous sialotorrhoea. $^{3}$ There will be a history of clear watery discharge form the EAC. This fluid will test positive for amylase.

A differential for this type of lesion is the first branchial cleft cyst. These cysts can be divided into two types. Type I lesions present in the parotid region and are ectodermal in origin; they appear clinically as soft cysts lined by squamous epithelium. Type II lesions present as a cyst, sinus, fistula, or any combination, and are of ectodermal and mesodermal origin, containing either skin appendages or cartilage., ${ }^{4,5}$

In this case, the palm leaf spine appears to have pierced through the EAC into the parotid thereby opening up a pathway for the spread of infection. This was evidenced intraoperatively by the trajectory of the opening of the fistula in relation to the fibrotic mass. Correct diagnosis and appropriate surgical intervention are important to minimise the incidence of recurrence and injury to the facial nerve. ${ }^{6,7}$

The fibrotic mass which was a focus of inflammation (as evidenced by the recurrent episodes of parotid swelling and otorrhoea) was most probably the retained spike of the leaf. Histological examination of the excised fibrotic mass did not show any plant material. It was most likely consumed by the foreign body reaction.

\section{Conflict of interest}

The authors declare no conflict of interest.

\section{Acknowledgements}

The authors would like to thank Dr J Ogonowski for his assistance.

\section{ORCID}

J Joseph (iD https://orcid.org/0000-0002-7356-7177 S Maharaj (iD https://orcid.org/0000-0002-2118-2400 


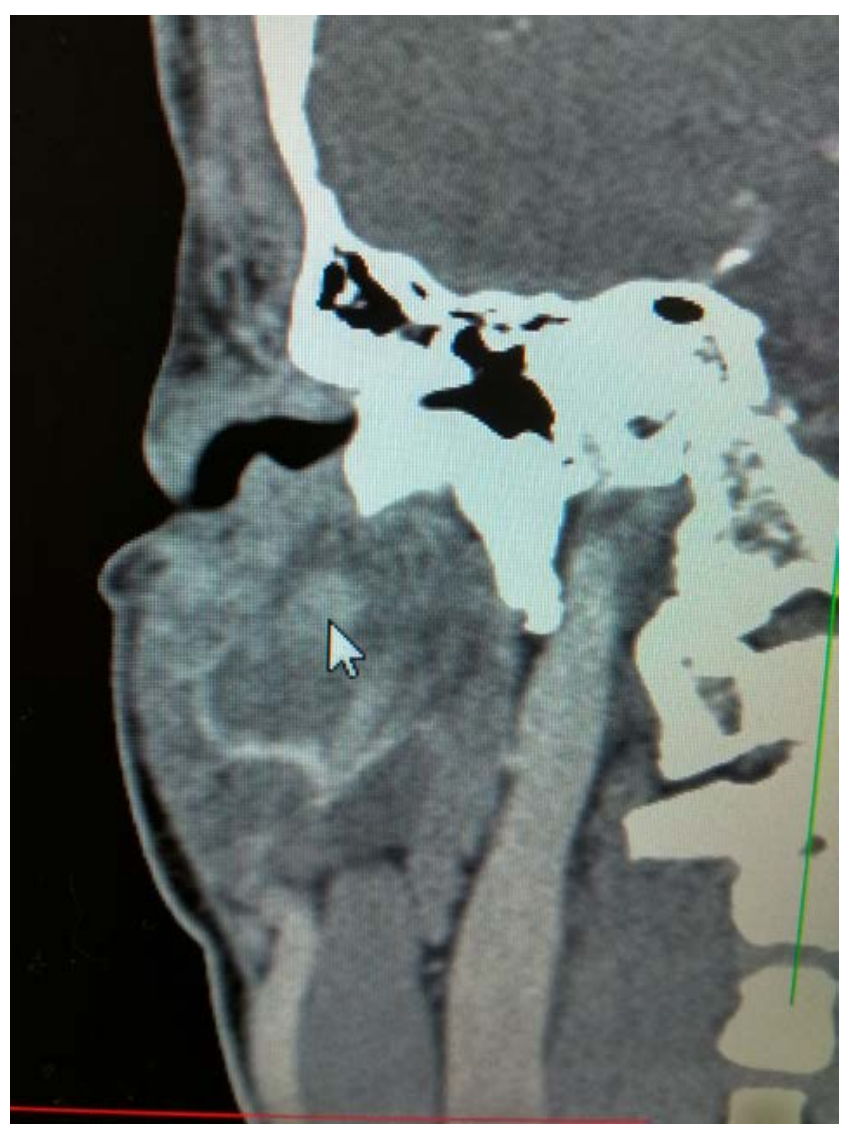

\section{REFERENCES}

1. Akinbami BO. Traumatic diseases of parotid gland and sequalae. Review of literature and case reports. Niger J Clin Pract. 2009;12(2):212-5.

2. Avery BS. A sialocoele and unusual parotid fistula - a case report. Br J Oral Surg. 1980;1:40-4.

3. Massimo F, Benfari G, Franco M, et al. Foramen of Huschke: case report and experimental procedure for the diagnosis of spontaneous salivary fistulae. J Oral Maxillofac Surg. 2009;67(8):1747-51.

4. Souza AR, Uppal HS, Zeitoun RD. Updating concepts of first branchial cleft defects: a literature review. Int J Pediatr Otorhinolaryngol. 2002;62:103-9.

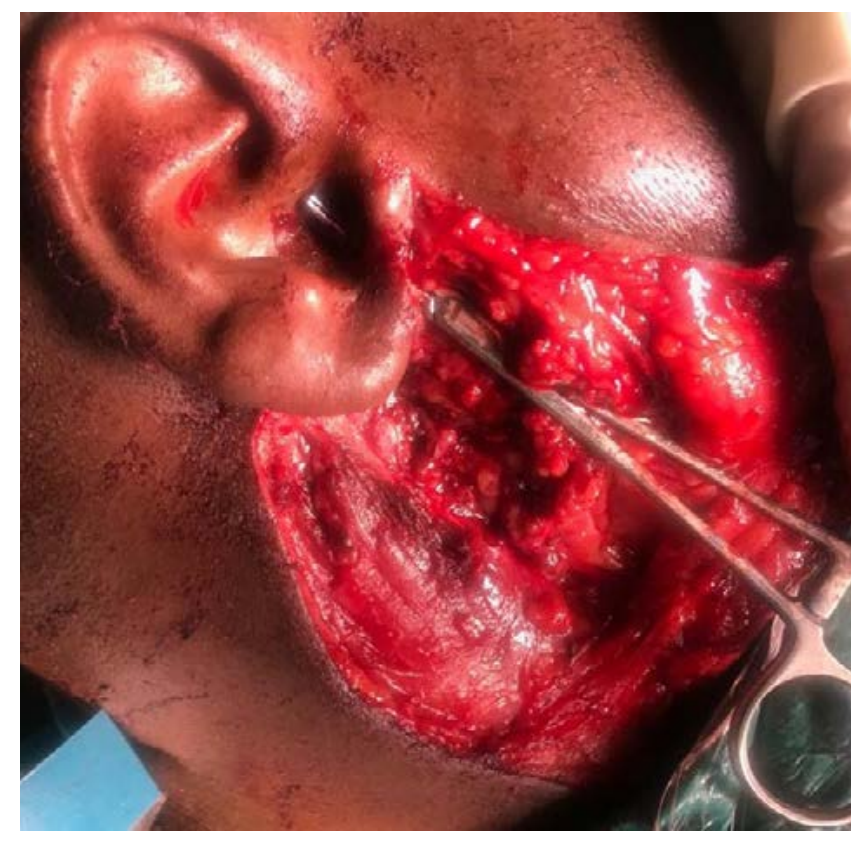

Figure 1: (Left) Right parotid mass (white arrow) seen on coronal CT scan

Figure 2: (Above) Intraoperative superficial parotidectomy with instrument demonstrating the fistula tract

5. Triglia JM, Nicollas R, Ducroz V, Koltai PJ, Garabedian EN. First branchial cleft anomalies: a study of 39 cases and a review of the literature. Arch Otolaryngol Head Neck Surg. 1998;124:291-5.

6. Kumar R, Sikka K, Sagar P, Kakkar A, Thakar A. First branchial cleft anomalies: avoiding the misdiagnosis. Indian J Otolaryngol Head Neck Surg. 2013;65:260-3.

7. Magdy EA, Ashram YA. First branchial cleft anomalies: presentation, variability and safe surgical management. Eur Arch Otorhinolaryngol. 2013;270:1917-25. 